\title{
Cognitive processing speed is high until age 60: Insights from Bayesian modeling in a one million sample (with a little help of deep learning)
}

\author{
Mischa von Krause ${ }^{1}$, Stefan T. Radev ${ }^{1}$, and Andreas Voss ${ }^{1}$ \\ ${ }^{1}$ Heidelberg University, Institute of Psychology
}

\section{- Abstract}

7 Processing speed is a fundamental aspect of human cognition and intelligence. Many studies from the

8 last decades report that processing speed, typically measured as mean reaction time in simple cognitive

9 tasks, significantly slows down in old age and already declines in young and middle adulthood. Our study employs a Bayesian diffusion model approach to disentangle different cognitive components involved in simple decision-making. We apply our model to a large data set of more than one million participants, which allows us to provide fine-grained and robust analyses of age differences. Since standard Bayesian methods are not suitable to data sets of this size, we use a novel deep learning method for parameter estimation. Our results indicate that processing speed is stable from young adulthood until an age of about 60 . The typical age-related slowdown in mean response times in this age range seems attributable to increases in decision caution and slower non-decisional processes - like encoding and motor response - but not to differences in cognitive processing speed. Our research has important implications for all fields concerned with age-related patterns in cognition and challenges widespread beliefs about the relationship between age and cognitive speed.

\section{Introduction}

Speed of information processing is a fundamental property of cognitive agents and an important prerequisite for timely and adequate responses in complex environments. Older people are often assumed to be slower thinkers than younger people - a notion that has significant consequences in work life $[1,2]$ and that has seemingly found strong empirical support. Over the past decades, a large body of research has consistently reported a negative relation between processing speed and age, that is, older people tend to be slower than younger people across a wide variety of cognitive tasks and contexts $[3,4]$. This approximately linear trend starts already in young adulthood, at ages 20 to $30[5,6,3,7]$, and has been reported in both cross-sectional and longitudinal studies $[8,7,3,9]$. The notion that processing speed already declines over young and middle adulthood has important implications for the study of human cognition. Since developmental patterns of cognitive abilities are linked to changes in the brain [10], studying the former can also provide insights into the neurophysiological basis of cognition.

The vast majority of findings on age and processing speed rely on mean response times (RTs) in elementary cognitive tasks (e.g., comparison of two letters) as a measure of basic speed of information processing $[4,5,11]$. However, this approach has two major shortcomings. First, the solitary use of mean RTs does not utilize the full information contained in empirical response time distributions and ignores accuracy data also obtainable from experimental paradigms. Second, mean RTs are not pure measures of processing speed, but instead represent the sum total of disparate cognitive processes [12]. For instance, speed-accuracy trade-offs (i.e., different settings of response caution that affect both speed and accuracy of responses) or the time taken up for encoding and motor processes contribute 
to the observed response time, although they are unrelated to cognitive processing speed. Thus, the extent to which mean RTs reflect processing speed is, at the very least, debatable [13, 14, 15].

Mathematical models of cognition strive to decompose behavior in interpretable and neurophysiologically plausible constructs. One of the most popular process models for explaining RT data is the diffusion model (DM, [16, 17, 18, 19, 20], see Materials and Methods section for a more detailed description of the model). By employing the DM, it is possible to obtain a process-pure estimate of processing speed through the model's drift rate parameter. This measure of processing speed is independent of decision caution (boundary separation) and the time required for encoding and motor processes (non-decision time). Moreover, the parameters of the DM have been extensively validated both experimentally $[21,22,23]$ and neurophysiologically $[24,25,26]$.

In the past two decades, a growing number of diffusion modeling studies on age differences in a great variety of experimental environments has been published $[23,27,28,29,30,31,32,14,33$, $34,35,36,37,38]$. Most of these studies compared groups of young adults, around age 20, with old adults, aged 65 and older, with respect to the model's parameters. Interestingly, it has often been reported that processing speed exhibits no differences between young and old adults. Conversely, decision caution and non-decision times were often markedly increased in old age.

Although model-based analyses of cognitive aging have many advantages over the direct analysis of raw data, many model-based studies have two serious shortcomings, both related to the samples used. First, sample sizes were comparably small in most studies, which is especially problematic for research on individual differences seeking to increase reliability through larger samples. For instance, a recent meta-analysis summarizing 25 studies had a total sample size of only 1,503 observations, indicating an average sample size of 60 participants per study [39]. Second, most studies only compared two age groups, typically college-age students and older adults aged 65 to 75 . Taken together, these two aspects severely limit the generalizability of previous results, especially with regard to the age span between 25 and 65 years, that is, large parts of young and middle adulthood.

There are two main reasons for the small sample sizes common for diffusion modeling studies. First, data collection for such studies is tedious, given the large number of trials per person that were long thought to be required for diffusion modeling [17]. However, such requirements are now considered as largely overstated [40,41]. Second, and more importantly, fitting the diffusion model to observed data is computationally expensive, especially when employing sampling-based Bayesian estimation methods. Thus, obtaining individual parameters even from moderately large samples is often infeasible for practical reasons. Yet, in order to provide a robust analysis of individual differences in processing speed in relation to age, a rather large data set including participants across the entire lifespan seems imperative.

In recent years, Bayesian methods have become the gold-standard for model-based inference in cognitive modeling [42]. Bayesian methods allow for principled uncertainty quantification in the form of full posterior distributions over quantities of interest (e.g., the parameters of a cognitive model). Once estimated, the posterior distribution can be used to extract credibility intervals or to perform posterior predictions to assess the quality of model fit. Moreover, posterior correlations between model parameters can be extracted and used as a measure of (linear) disentanglement between parameters at an individual-level. However, a major disadvantage of standard Bayesian methods for cognitive models (e.g., Markov chain Monte Carlo methods) is their computational slowness, which makes them impractical or even impossible to apply in data-rich contexts. In this work, we therefore demonstrate the utility of a novel deep-learning framework developed to scale up model-based Bayesian inference to millions of data sets [43].

We present an analysis of cross-sectional age differences in diffusion model parameters estimated from a massive data set $(N>1,000,000)$, utilizing response times and accuracy rates collected in an online implicit association test ([44]). Notably, this sample is multiple orders of magnitude larger than the samples used in all previous diffusion model studies combined. Our deep-learning architecture for parameter estimation is based on a a two-stage inference framework which is illustrated and described in the Materials and Methods section [43]. Regarding chronological age, our sample covers childhood till late adulthood (ages 10 to 80), with a sufficient depth for fine-grained and robust year-by-year analysis.

In our study, we derive substantial insights into individual differences in cognitive parameters 
by applying Bayesian diffusion modeling to a large sample with the help of modern deep learning methods. Accordingly, our approach yields unique and robust findings on age-related patterns of different aspects of cognition, separating processing speed, decision caution, and non-decision parts of response times.

We observe a clear non-linear association between drift rate (as an index of processing speed) and age, which is strikingly different than the one implied by mean RTs and far more informative than the age differences found in previous diffusion model studies. Thus, our model-based analysis reveals a picture of age differences in cognitive parameters yielding a radically different implication than the one based on analyses of raw RT data.

\section{Results}

Figure 1 depicts our main findings. Mean correct response times, processing speed, decision caution, and correct non-decision time are plotted against age in years. The figure shows results for one of the two experimental conditions (incongruent trials). The other condition (congruent trials) yields very similar patterns, which are presented in the SI Appendix. Each dot represents the mean of the individual posterior parameter means for one year of age. The vertical bars represent one standard deviation within each year of age. Descriptive statistics for all parameters can be found in the SI Appendix. To better describe the age-related patterns we found, we estimated linear Bayesian change-point models combined with piece-wise Bayesian ridge regressions (see Materials and Methods). The estimated change points and piece-wise regression lines together with their respective uncertainties are also depicted in Figure 1.

As evident from Figure 1, cross-sectional mean correct RTs decrease sharply from the age of 10 to about 20, with the change point regarding the age trend estimated at age 19. After that, mean correct RTs show a quasi-linear increasing trend until the estimated change point of age 62 . Thereafter, the average increase in response times per year accelerates, although data become more sparse when approaching age 80 (e.g., $n=169$ for age 80).

Drift rates, that is, our proxy for processing speed, increase notably from age 10 to 30 in our cross-sectional data. After this, mean levels in drift rates remain fairly stable until an age of 60 , showing little age-related differences during middle adulthood. At around the age of 60 , an accelerated negative trend in cognitive processing speed commences, which holds until an age of 80. Importantly, this inverted $U$-shaped pattern does not mirror the age trends found for the other diffusion model parameters or mean RTs. Our change points are estimated at ages 25 and 60 . The change point model misses the minor increase in drift rates that continues until age 30, as well as the slight decrease in drift rates starting at age 50 .

Boundary separation, that is, estimates of decision caution, decrease from age 10 to about age 20, after which they show a quasi-linear increase until an age of 65 . Thereafter, the average increase in response times per year accelerates. Change points are estimated at ages 18 and 65 . It should be noted that in the congruent experimental condition (see SI Appendix), the change point for boundary separation was already estimated at age 40 , and the subsequent increasing trend was less pronounced there.

Finally, non-decision time estimates, that is, the time taken for encoding and motor response, decrease from age 10 to the estimated change point of age 15, after which show a quasi-linear increase until an age of 80 . The age differences for decision caution and non-decision times closely mirror the pattern found for RTs, suggesting that these components could have a large impact on the mean levels of response latencies over the life course.

As can further be observed, variability in mean correct RTs increases across the lifespan. The trend is paralleled by the increase in variance found for non-decision times. Conversely, the between-person variability for boundary separation and drift rates shows no age-related increase.

In order to ensure that our findings hold across a wide range of conditions, we conducted several robustness checks. Figure 2 shows that the mean level trend for drift rates is robust across genders, levels of education, and experimental conditions (congruent vs. incongruent). However, the increased decline in drift rates after age 60 is more pronounced for the incongruent condition, and women show 

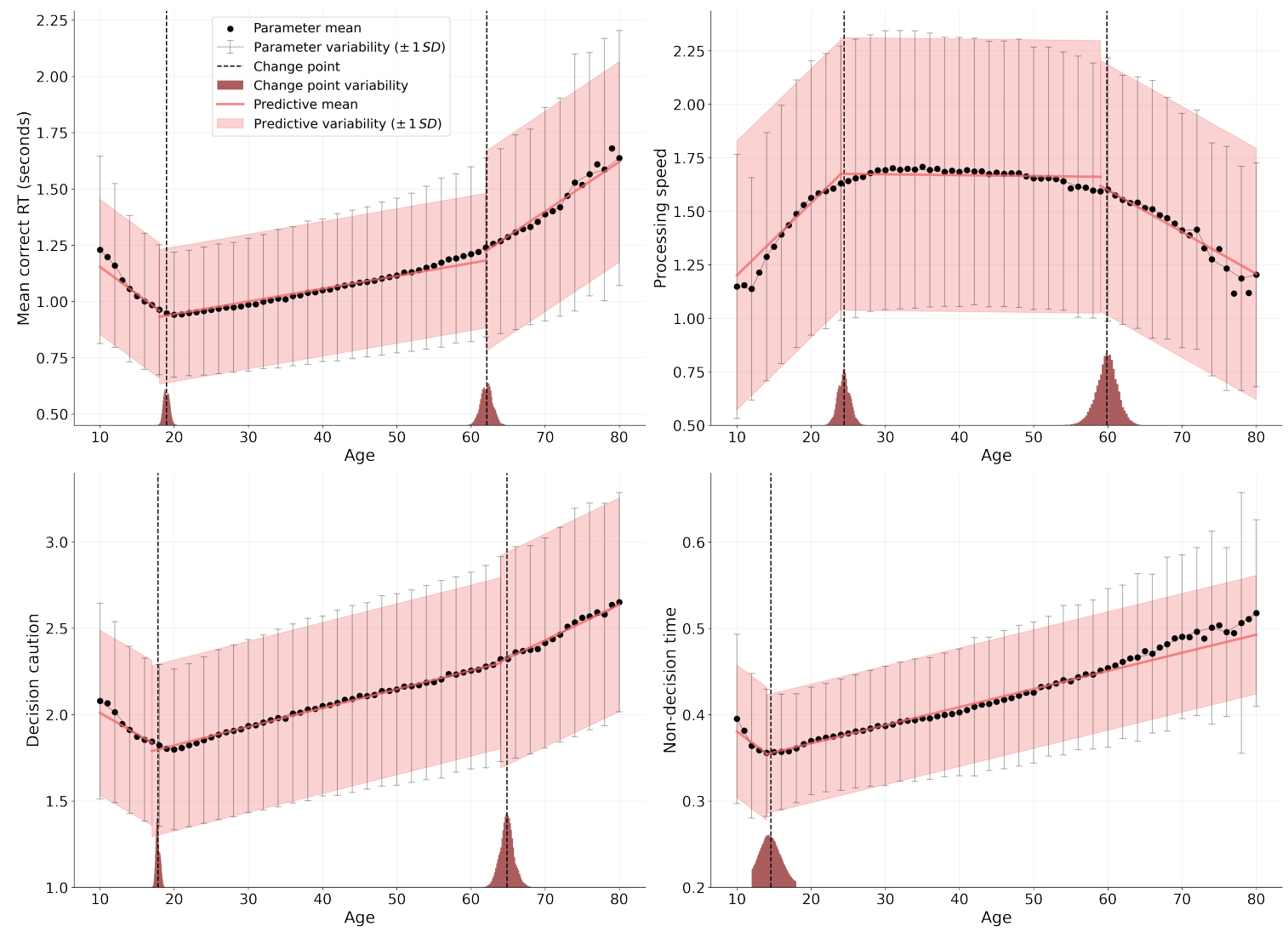

Figure 1: Mean correct response times (RTs) and diffusion model parameters as a function of age. Black points indicate means computed separately for each year of age. Bars indicate standard deviations (only shown for every second year for better clarity). Red lines denote the Bayesian piece-wise ridge regression model's mean predictions, which describe the observed means fairly well. The shaded red region denotes the uncertainty (standard deviation) of the piece-wise model's predictions. The dashed lines indicate the mean change points estimated from the per-age-group averaged data, with the full posterior distributions (scaled for readability) of the change points shown at the bottom of each plot. Both the data- and model-implied standard deviations highlight the great variability within each year of age. Nevertheless, the year-specific means suggest a clear and consistent pattern for mean correct RT and each parameter. The figure depicts drift rates and boundary separations for the incongruent condition and non-decision times obtained from correct responses. Very similar trends for the congruent condition and non-decision times from incorrect responses can be found in the SI Appendix. 

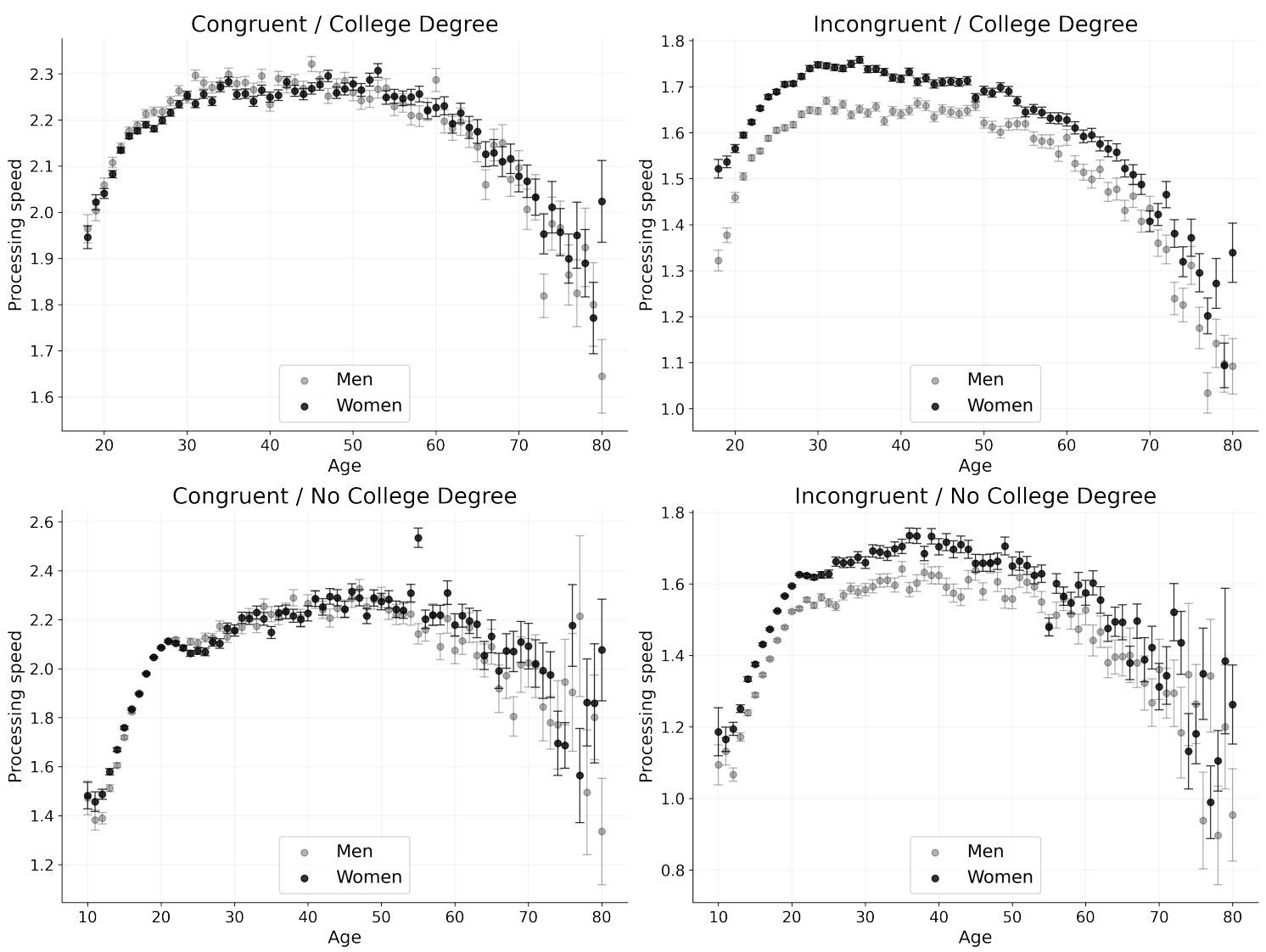

Figure 2: Processing speed as a function of age, experimental condition, and demographic variables. We observe the same inverted $U$-shape as in our main analysis. Vertical bars denote standard errors of the mean (SEMs). 
higher mean levels of drift rates also in the incongruent condition. The vertical bars in Figure 2 indicate standard errors of the means. Due to the very large sample size, standard errors are very small for all age groups except for the very old participants. This guarantees that the differences in processing speed across the lifespan were assessed very accurately. We performed additional robustness checks by comparing the trends in age effects across different sub-samples. For this purpose, we first divided the sample into four almost evenly sized sub-samples. Across these sub-samples, mean-level patters were virtually identical. The same was true when comparing participants born in the United States with those originating from other countries, as well as for the comparison between participants working on tasks with different classes of stimuli (i.e., "Black/White" or "African American/European American"). All these additional analyses can be found in the SI Appendix where we also report correlations between the different diffusion model parameters, both across participants and within each person - the latter by utilizing the individual posterior distributions.

\section{Discussion}

In this work, we presented a cross-sectional study of age differences in mean response times and cognitive processes as measured by the diffusion model. We applied the diffusion model to a massive data set, containing response time and accuracy data from the implicit association test (IAT). Our sample covers large parts of the human lifespan (ages 10 to 80) in sufficient depth for a fine-grained analysis of age differences at a year-specific level. Given the sample size, our analyses would have been infeasible using standard parameter estimation procedures. Thus, the novel deep learning approach utilized in this work for parameter estimation was both necessary and extremely efficient for the task at hand. Our findings stand in pronounced contrast to previous findings on age differences in processing speed. The implications of our work are thus relevant for all research domains studying cognitive development across the lifespan. We will now discuss the implications of our findings in turn.

\section{Mean response times}

Our results replicate the age-related decline in mean response times previously reported $[5,6,3,7,4]$. In our sample, mean response times showed a negative trend during the teenage years, were fastest around age 20, and showed a nearly linear increase thereafter, which further accelerated after the age of 60 . It is important to note that these findings are in line with earlier response time studies. This indicates that the diverging patterns found for the diffusion model parameters are not based on qualitatively different raw data than previously collected in the field.

\section{Decision caution}

In the diffusion model, decision caution, that is, the amount of information sampled before making a decision, is represented by the boundary separation parameter [18]. Our results suggest that, on average, boundary separation declines from age 10 to approximately age 18 , indicating that people at college age were the least cautious in our sample - they were most willing to trade off accuracy for speed. After age 18, decision caution increases linearly until about age 65 in the incongruent condition, with a greater increase per year thereafter until age 80 . In the congruent condition, the increasing trend in decision caution during old age was less pronounced and the change in age-related trends was estimated to occur already at age 40. Both findings might be attributable to the lower task difficulty in the congruent condition. Moreover, the trend towards higher decision cautiousness becomes noticeable already very early in adult life. Thus, the increase in the amount of information sampled before making a decision provides a first explanation for the age-related increase in RTs starting in young adulthood. 


\section{Encoding and motor processes}

Non-decision time is the diffusion model parameter that represents all processes beyond information sampling and evidence accumulation in a decision task. These processes are typically thought to encompass the time taken for encoding of stimuli and motor response execution [45, 22]. Interestingly, in our sample, non-decision times were, on average, fastest around age 14 to 16, with people outside this range needing more time for non-decision processes. It seems that these processes, should they represent a trait-like ability, reach their peak earliest among all cognitive abilities typically studied in the literature [7]. After age 16, non-decision times exhibit a linear increase that continues up until age 80. Thus, the increase in the time needed for non-decision processes provides a second explanation for the slower RTs found with increasing age, already among young adults and in middle adulthood.

\section{Processing speed}

Our most significant finding concerns the drift rate, that is, the parameter representing processing speed in the diffusion model framework. The drift rate denotes the average rate of information sampling per time unit and, theoretically, represents a process-pure measure, because speed-accuracy trade-offs and non-decision aspects have been controlled for by the other two main diffusion model parameters. During early adulthood, drift rates showed, on average, a continuous positive age trend, that is, processing speed became faster from age 10 to age 30. Processing speed thus peaks notably later than the lowest points of decision caution (around age 20) and non-decision times (around age 15). This result partly mirrors previous findings reporting that processing speed is still high around age 30 [6]. Yet, in our sample, processing speed showed a slight increase from the age of 20 to 30 , which is in contrast to previous findings based on the analysis of mean RTs. It should be noted that our change point analysis indicated that the positive age trend in drift rates is weaker from age 25 to age 30 than in the years before that, with the corresponding change point estimated at age 25 .

Most importantly, our analyses suggest that the average levels of processing speed remain roughly stable across the entire middle adulthood (age 30 to 60), with only slight decreases from age 50 on. This surprising finding remains hidden if only mean response times are analyzed, as these do not reflect a pure measure of cognitive processing speed, but are heavily influenced by decision caution and time required for motor processes. The pattern was robust across different stimuli, experimental conditions, and several demographic factors. Accordingly, we conclude that the age-related increase of RTs in early and middle adulthood can be attributed exclusively to differences in decision caution and non-decision time, not to differences in processing speed. Only after about age 60, drift rates start to show an accelerating negative age-related decline, with the lowest mean values found for the oldest participants. These age-related declines in processing speed in old age are in line with what has been reported in previous studies on cognitive aging. However, our analysis suggests that the decline starts much later in life than has typically been assumed.

\section{Main Discussion}

The higher boundary separations, non-decision times, and lower drift rates found for people aged 60 and older jointly explain the accelerated age-related increase in mean RTs among the oldest participants. From about age 60 on, these three components contributing jointly to mean RTs all show age trends that lead to slower RTs. In other words, older people display higher decision caution, slower non-decision time, and slower processing speed.

Our key findings also explain the age-related findings reported in previous diffusion modeling studies. Typically, these studies compared two groups of participants: college-aged students and people aged 65 and older $[23,27,28,29,30,31,32,14,33,34,35,38]$. A consistent result of these studies was that older participants show higher boundary separations and non-decision times, but comparable drift rates.

When looking at our data, it is plausible that the linear age trends from age 20 onward we found for boundary separation and non-decision times are consistent with the effects found in previous two-group studies. However, previous studies reporting no differences in drift rates between young 
and late adulthood might have overlooked the non-linear age trend and the peak of drift rates from the age of 30 to age 50, because this group was not represented in the samples.

Our results are also in line with recently reported results on age differences in diffusion model parameters using a continuous assessment of age [36]. In this study, for a wide variety of different tasks a peak in processing speed around age 30 was also observed. However, sample size across later young adulthood and middle adulthood was too small to reveal clear age trends.

Another interesting finding emerging from our study is the fact that diffusion model parameters showed different cross-sectional patterns of across-person variability over the lifespan. While the variances of boundary separation and drift remained roughly the same even into old age, non-decision times showed an increase in variability after the age of 60 . The latter pattern is also present for mean RTs. Thus, it seems plausible that the greater spread in mean RTs observed for older people is attributable to greater inter-individual differences in encoding and motor processes, not in processing speed or decision caution.

Finally, this is one of the first studies to report age differences in diffusion model parameters in late childhood and adolescence (but see [46]), thus allowing the study of differentiable temporal patterns in these age periods. Most notably, the fastest non-decision times were observed already at ages 14 to 16, with mean RTs, processing speed, and decision caution all showing much later turning points.

The differing age-related patterns of the diffusion model parameters become more plausible when viewed in the context of the literature linking changes in cognitive abilities with changes in their neurophysiological basis [10]. According to the Scaffolding Theory of Aging and Cognition (STAC-r; [47]), people differ in their use of different compensatory techniques (e.g., activation of additional neural networks), all of which aim to counter the detrimental effects of age-related changes in brain structure. While such compensatory strategies might be well-suited to keep the level of processing speed in simple decision-making tasks high across large parts of the lifespan, more basal processes such as the ones captured in non-decision time might be less adaptable [36].

Our study has a number of advantages to previous studies of cognitive aging, the most prominent being i) the massive sample size allowing for detailed age-related analyses and ii) the use of Bayesian diffusion modeling to disentangle different components of the decision process in a robust and theoretically grounded way. However, we must also note some limitations of this study.

First, the data used here comes from only one particular type of decision-making task, namely the race IAT. One might thus question whether our results generalize to other experimental paradigms or real-life scenarios. Regarding this limitation, it should be noted, that our results i) replicated across different experimental conditions and types of stimuli and ii) were in line with the findings reported in a number of studies on age-differences in diffusion model parameters. These previous studies spanned a vast variety of experimental tasks and paradigms, although with much smaller sample sizes. Thus, it seems plausible that our results, albeit based on a single type of task, should generalize to many other typical decision-making contexts.

A second limitation concerns the cross-sectional nature of our findings. Thus, it remains an open question whether the age differences and trends found in our data represent within-person developmental processes. We did not study longitudinal change, and neither did we account for cohort effects. However, given the clear age trends (with the majority of means almost perfectly aligned across age groups) found for the cognitive parameters of interest, we argue that our data provide as clear a picture of developmental patterns as is reasonably achievable using cross-sectional data. We also want to note that the IAT data made publicly available by Project Implicit [44] includes data-sets from the years 2002 to 2020, thus making it possible to study cohort effects, and also participant IDs, making it possible to study longitudinal change in participants taking the task several times. Such analyses were beyond the scope of this paper, but might be well worthwhile in future endeavours.

For anyone interested in replicating or expanding our analyses using similar data and estimation methods, we provide open source code and pre-trained deep learning networks for preprocessing and obtaining Bayesian diffusion model parameter estimates on our GitHub page (https://github.com/stefanradev93/DataSizeMatters).

To conclude, according to our analysis of a large data set of human response times, processing 
speed increases until the age of 30 , remains at a plateau until an age of around 60 , and declines thereafter. Furthermore, the slowdown in mean response times found already in young and middle adulthood seems attributable to age-related changes in decision caution and non-decision times. Only in old age, cumulative effects of all three cognitive parameters - processing speed, decision caution, non-decision times - contribute to an accelerated slowdown that is also evident from the raw RT data. Thus, for large parts of the human lifespan and typical work careers, our results challenge the widespread notion of an age-related slowdown in cognitive speed.

\section{Materials and Methods}

Our analyses are based on publicly available race IAT data provided by Project Implicit [44]. We extracted raw response time and accuracy data, as well as demographics, all of which were collected from September 2016 to December 2018. All data are openly available on the Project Implicit OSF page: https://osf.io/y9hiq/. In addition, all analysis scripts for reproducing the results are available at: https://github.com/stefanradev93/DataSizeMatters

\section{Participants}

Our original sample contained 1,804,325 people. We excluded cases that did not complete the task, did not provide their year of birth, were older than 80 or younger than 10 years at time of data collection, had more than $10 \%$ response times under $300 \mathrm{~ms}$, or had below change accuracy. In order to be able to obtain full data-informed parameter sets for each person, including the error non-decision time, we excluded all participants with $100 \%$ accuracy. Further, we excluded trials faster than $300 \mathrm{~ms}$ or slower than 10 seconds. After fitting the diffusion models, we excluded cases with estimates for drift rates, boundary separations, or non-decision times beyond the borders of our respective (very broad) prior ranges (see below: The diffusion model). This left us with a final sample of $1,185,882$ people. Of these, $38.69 \%$ were female and $61.30 \%$ were male (the question asked was about the sex assigned at birth). Mean age was 27.42 years $(S D=12.33)$, with a robust sample size across the entire age span of 10 to 80 years. About halve of the participants (46.89\%) had completed at least college level education. The majority $(84.06 \%)$ of the participants indicated that they were born in the USA, with the rest reporting different countries of origin.

\section{Task}

The race IAT is a quasi-standard cognitive task originally designed to measure implicit racial bias [48]. In a series of binary decisions, people have to classify words and images as belonging to one of two categories, for example "good/bad" or "Black person/White person". Across the two different main blocks of the experiment, the mappings of the categories to the same response button change. "Good" might share a common response key (e.g., left) with "Black person" in the first condition, and then be paired with "White person" in the second condition. 60 trials are completed in each of the two conditions. The difference in mean response times is then used to obtain a measure of implicit bias [49]. The exact procedure and materials can be found on the Project implicit OSF page (https://osf.io/y9hiq/, [44]). We did not use the IAT as an instrument to study implicit cognition, but instead as an example of a simple binary decision task.

\section{The diffusion model}

In the present work, we employ the diffusion model (DM), a prominent mechanistic model of neurocognitive dynamics designed to explain human performance in simple decision-making tasks [18]. The DM is embedded in the larger model class of evidence accumulator models (EAMs), which conceptualize information processing as a gradual, temporally-ordered, and noisy process [50]. A core assumption of the DM is that task-relevant information is integrated at multiple neurocognitive levels in which sensory evidence for one of the alternatives is dynamically accumulated at a constant rate. A categorical decision for one of the alternatives is determined as soon as a pre-defined threshold 

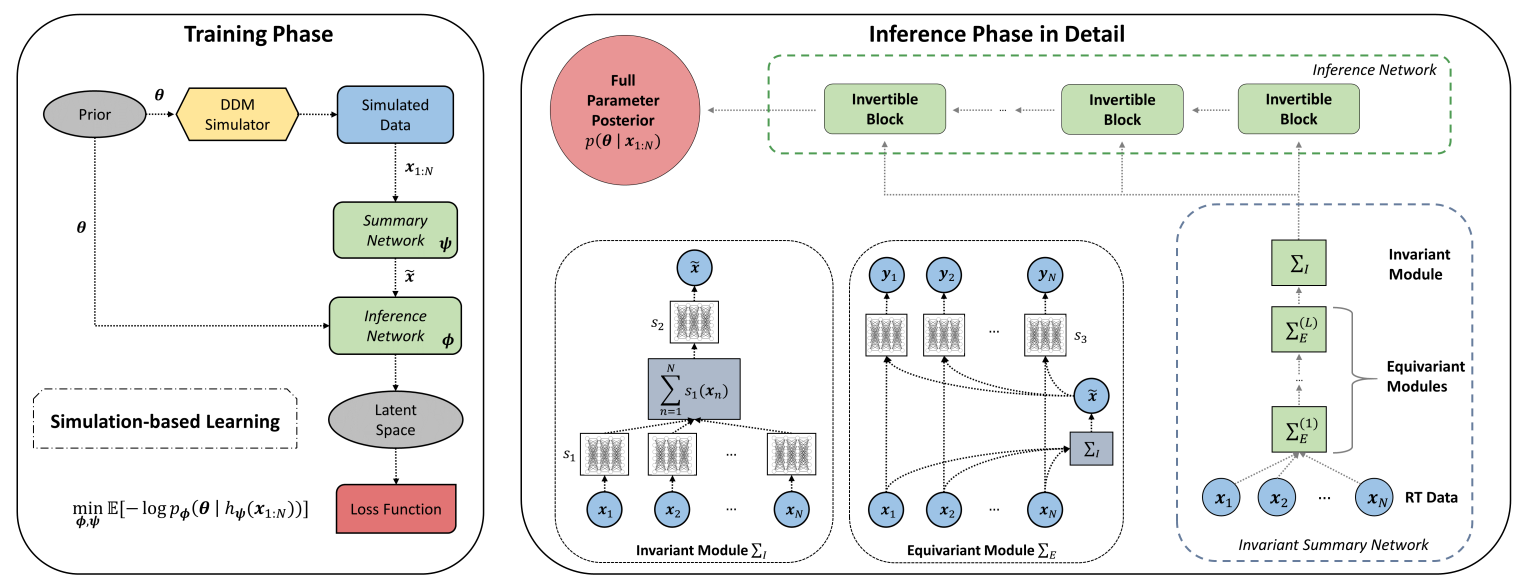

Figure 3: The BayesFlow framework used for individual parameter estimation on more than a million data sets. Left panel: During training, the computational model serves as an "instructor", which, by means of simulations, guides the summary $(\boldsymbol{\psi})$ and the inference network $(\phi)$ to become "experts" in inverting the model and recovering plausible estimates of cognitive parameters $(\boldsymbol{\theta})$. Right panel: During inference, the trained networks efficiently process each observed data and estimate the full posterior over parameters of interest. The training effort thus "amortizes" over multiple estimation passes, as no further training of the networks is required. Specialized invariant and equivariant networks [53] are required for processing the i.i.d. response times and accuracy data obtained in each IAT experiment.

is reached. Moreover, the key parameters of the DM are well-validated in experimental settings $[21,22,23]$ and well-grounded in biological neural-network theory [50].

In order to decompose performance in the race IAT into meaningful cognitive constructs, we formulate and fit a DM with six parameters: $\boldsymbol{\theta}=\left(v_{1}, v_{2}, a_{1}, a_{2}, \tau_{c}, \tau_{n}\right)$. Here, $v_{1}$ and $v_{2}$ denote the speed of information processing (drift rates) in the two experimental conditions; $a_{1}$ and $a_{2}$ denote the decision thresholds (boundary separation); $\tau_{c}$ and $\tau_{n}$ denote the additive non-decision time constants for correct and incorrect responses, respectively. We estimate separate drift rates and boundary separations for the congruent and incongruent conditions, because these parameters have been shown to differ across the IAT conditions in previous studies [51]. We estimate separate non-decision times for correct and incorrect trials due to the nature of the race IAT task (i.e., trials do not terminate immediately following a wrong response but require an additional response from the participants).

Our choice of Bayesian priors for the DM parameters reflects the goal to cover meaningful parameter ranges, as known from previous studies [52]. However, we also place uniform priors over the plausible numerical ranges in order to render the data maximally informative for posterior inference. We place broad uniform priors over both drift rates, that is, $v \sim \mathcal{U}(0.1,7)$, which we deem sufficient to cover the entire range of realistically observable processing speeds. On the basis of similar considerations, we place a broad uniform prior over the boundary separation parameters, $a \sim \mathcal{U}(0.1,4)$. For the non-decision constants, we use $\tau_{c} \sim \mathcal{U}(0.1,3)$ and $\tau_{n} \sim \mathcal{U}(0.1,7)$, incorporating our expectation of longer non-decision times for incorrect responses in the particular task.

\section{Parameter estimation}

Performing Bayesian estimation on hundreds of thousands participants is not feasible with current gold-standard Markov chain Monte Carlo (MCMC) methods. We therefore resort to amortized Bayesian inference via specialized neural networks, which nevertheless guarantee correct posterior inference under perfect convergence [43]. The term amortized inference refers to an approach which reduces the computational cost of Bayesian estimation by splitting the analysis into a costly upfront training phase, followed by an extremely efficient inference phase [43]. Figure 3 depicts our parameter estimation procedure. 
Basically, the BayesFlow method comprises a summary network $h$ and an inference network $f$ which are trained jointly via simulations from the full Bayesian model:

$$
p\left(\boldsymbol{\theta}, \boldsymbol{x}_{1}, \ldots, \boldsymbol{x}_{N}\right)=p(\boldsymbol{\theta}) \prod_{n=1}^{N} p\left(\boldsymbol{x}_{n} \mid \boldsymbol{\theta}\right)
$$

Simulations are realized via a Monte-Carlo stimulation program which efficiently samples from the prior and runs the DM with the sampled parameter configurations to generate synthetic data sets. The outputs of the simulation program are then fed to the neural networks and the networks' parameters are optimized via standard backpropagation. The role of the summary network is to reduce data sets of arbitrary size to fixed-size vector representations in a completely end-to-end manner. The role of the inference network is to generate samples from an approximate posterior $p_{\phi}$ via a conditional invertible neural network (cINN) $f_{\phi}$. Thus, once trained, the two networks are able to efficiently approximate the true posterior $p\left(\boldsymbol{\theta} \mid \boldsymbol{x}_{1: N}\right)$ given any possible data set arising from the model. Complete inference using the BayesFlow framework is illustrated in Figure 3.

Denoting the inference network parameters as $\phi$ and those of the summary networks as $\psi$, the two networks are trained to minimize the following Kullback-Leibler (KL) divergence criterion:

$$
\min _{\boldsymbol{\phi}, \boldsymbol{\psi}} \mathbb{E}_{p(\boldsymbol{\theta}, \boldsymbol{x})}\left[-\log p_{\boldsymbol{\phi}}\left(\boldsymbol{\theta} \mid h_{\boldsymbol{\psi}}\left(\boldsymbol{x}_{1: N}\right)\right)\right]
$$

which corresponds to minimizing the discrepancy between the true and the approximate amortized posterior induced by the networks. To train the networks, we performed approximately 50000 simulations from the DM model with the priors for the parameters as described in the previous paragraph. Training the networks took approximately 8 hours on a GPU-accelerated laptop. Inference on the entire data set took approximately 24 hours on a machine without GPU-acceleration.

\section{Bayesian Workflow}

To further enhance the transparency and trustworthiness of our Bayesian pipeline, we follow the steps pertaining to a principled Bayesian workflow, as advocated by [54]. Accordingly, we partition our pipeline into the following steps: i) prior predictive checks; ii) checks of computational faithfulness; iii) checks of model adequacy/sensitivity; iv) posterior predictive checks. These validation results, along other robustness analyses, are described and visualized in the SI Appendix.

\section{Curve fitting}

Given the massive data set available for data mining, inferential statistics were of minor importance to our analyses. Due to the non-linear age-related patterns of cognitive parameters, we computed separate piece-wise Bayesian ridge regressions of each quantity of interest (mean correct RT and DM parameters) on age as the simplest and yet reasonable approximation of the observed age trends.

Our statistical analyses followed a two-step approach. First, we performed a linear Bayesian change-point regression on the age-group averaged data using the $R$-package for multiple change points $m c p$ [55]. Note, that this step ignores all variability within an age group and thus focuses on fast change point detection, which otherwise would have been infeasible if executed on the full data. In a second step, we extracted the posterior distributions of each change points and used the corresponding posterior means for a piece-wise Bayesian ridge regression on the full data set. In this way, the piece-wise model's predictive means and uncertainty account for the full variability in the estimated parameters.

We placed the following priors over change points to broadly reflect the trends visible in the data: mean correct response times - $t_{1} \sim \mathcal{U}(15,25), t_{2} \sim \mathcal{U}(35,70)$; drift rates $-t_{1} \sim \mathcal{U}(20,40)$, $t_{2} \sim \mathcal{U}(35,70)$; boundary separations - $t_{1} \sim \mathcal{U}(15,25), t_{2} \sim \mathcal{U}(35,70) ;$ non-decision times - $t_{1} \sim$ $\mathcal{U}(12,18)$, where the scales of measurement correspond to chronological age. For the Bayesian ridge regression, we used the default priors available through the scikit-learn implementation in the Python programming language. 


\section{Author Contributions}

Conceptualization, M.v.K; methodology, M.v.K. and S.T.R; formal analysis, M.v.K. and S.T.R.; investigation, M.v.K..; data curation, M.v.K. and S.T.R.; writing - original draft preparation, M.v.K. and S.T.R.; writing - review and editing, M.v.K., S.T.R, and A.V.; visualization, S.T.R. and M.v.K; supervision, A.V. All authors have read and agreed to the final version of the manuscript.

\section{Acknowledgements}

This research was supported by a grant from the German Research Foundation to the Graduate School 530 SMiP (GRK 2277; Statistical Modeling in Psychology).

\section{References}

[1] A. H. R. Commission et al., "National prevalence survey of age discrimination in the workplace $2015, " 2015$.

[2] J. T. Erber and B. A. Long, "Perceptions of forgetful and slow employees: Does age matter?," The Journals of Gerontology Series B: Psychological Sciences and Social Sciences, vol. 61, no. 6 , pp. P333-P339, 2006.

[3] T. A. Salthouse, "Selective review of cognitive aging," Journal of the International Neuropsychological Society: JINS, vol. 16, no. 5, p. 754, 2010.

[4] A. R. Jensen, Clocking the mind: Mental chronometry and individual differences. Elsevier, 2006.

[5] T. A. Salthouse, "The processing-speed theory of adult age differences in cognition.," Psychological review, vol. 103, no. 3, p. 403, 1996.

[6] T. A. Salthouse, "What and when of cognitive aging," Current directions in psychological science, vol. 13, no. 4, pp. 140-144, 2004.

[7] J. K. Hartshorne and L. T. Germine, "When does cognitive functioning peak? the asynchronous rise and fall of different cognitive abilities across the life span," Psychological science, vol. 26, no. 4, pp. 433-443, 2015.

[8] K. W. Schaie, "What can we learn from longitudinal studies of adult development?," Research in human development, vol. 2, no. 3, pp. 133-158, 2005.

[9] D. Zimprich and M. Martin, "Can longitudinal changes in processing speed explain longitudinal age changes in fluid intelligence?," Psychology and aging, vol. 17, no. 4, p. 690, 2002.

[10] J. Oschwald, S. Guye, F. Liem, P. Rast, S. Willis, C. Röcke, L. Jäncke, M. Martin, and S. Mérillat, "Brain structure and cognitive ability in healthy aging: a review on longitudinal correlated change," Reviews in the Neurosciences, vol. 31, no. 1, pp. 1-57, 2019.

[11] G. T. Frischkorn and A.-L. Schubert, "Cognitive models in intelligence research: Advantages and recommendations for their application," Journal of Intelligence, vol. 6, no. 3, p. 34, 2018.

[12] R. G. Pachella, "The interpretation of reaction time in information processing research," tech. rep., Michigan University Ann Arbor Human Performance Center, 1973.

[13] A.-L. Schubert and G. T. Frischkorn, "Neurocognitive psychometrics of intelligence: How measurement advancements unveiled the role of mental speed in intelligence differences," Current Directions in Psychological Science, vol. 29, no. 2, pp. 140-146, 2020.

[14] R. Ratcliff, A. Thapar, and G. McKoon, "Individual differences, aging, and iq in two-choice tasks," Cognitive psychology, vol. 60, no. 3, pp. 127-157, 2010. 
[15] V. Lerche, M. von Krause, A. Voss, G. T. Frischkorn, A.-L. Schubert, and D. Hagemann, "Diffusion modeling and intelligence: Drift rates show both domain-general and domain-specific relations with intelligence," Journal of Experimental Psychology: General, vol. 1, no. 1, 2020.

[16] R. Ratcliff, "A theory of memory retrieval.," Psychological review, vol. 85, no. 2, p. 59, 1978.

[17] R. Ratcliff and G. McKoon, "The diffusion decision model: theory and data for two-choice decision tasks," Neural computation, vol. 20, no. 4, pp. 873-922, 2008.

[18] R. Ratcliff and J. N. Rouder, "Modeling response times for two-choice decisions," Psychological science, vol. 9, no. 5, pp. 347-356, 1998.

[19] A. Voss, M. Nagler, and V. Lerche, "Diffusion models in experimental psychology: A practical introduction.," Experimental psychology, vol. 60, no. 6, p. 385, 2013.

[20] D. Fudenberg, W. Newey, P. Strack, and T. Strzalecki, "Testing the drift-diffusion model," Proceedings of the National Academy of Sciences, vol. 117, no. 52, pp. 33141-33148, 2020.

[21] V. Lerche and A. Voss, "Experimental validation of the diffusion model based on a slow response time paradigm," Psychological research, vol. 83, no. 6, pp. 1194-1209, 2019.

[22] A. Voss, K. Rothermund, and J. Voss, "Interpreting the parameters of the diffusion model: An empirical validation," Memory \&6 cognition, vol. 32, no. 7, pp. 1206-1220, 2004.

[23] N. R. Arnold, A. Bröder, and U. J. Bayen, "Empirical validation of the diffusion model for recognition memory and a comparison of parameter-estimation methods," Psychological research, vol. 79, no. 5, pp. 882-898, 2015.

[24] D. P. McGovern, A. Hayes, S. P. Kelly, and R. G. O'Connell, "Reconciling age-related changes in behavioural and neural indices of human perceptual decision-making," Nature human behaviour, vol. 2, no. 12, pp. 955-966, 2018.

[25] R. Ratcliff, Y. T. Hasegawa, R. P. Hasegawa, P. L. Smith, and M. A. Segraves, "Dual diffusion model for single-cell recording data from the superior colliculus in a brightness-discrimination task," Journal of neurophysiology, vol. 97, no. 2, pp. 1756-1774, 2007.

[26] S. Kühn, F. Schmiedek, B. Schott, R. Ratcliff, H.-J. Heinze, E. Düzel, U. Lindenberger, and M. Lövden, "Brain areas consistently linked to individual differences in perceptual decisionmaking in younger as well as older adults before and after training," Journal of Cognitive Neuroscience, vol. 23, no. 9, pp. 2147-2158, 2011.

[27] B. H. Ball and A. J. Aschenbrenner, "The importance of age-related differences in prospective memory: Evidence from diffusion model analyses," Psychonomic Bulletin \& Review, vol. 25, no. 3, pp. 1114-1122, 2018.

[28] J. Dully, D. P. McGovern, and R. G. O'Connell, "The impact of natural aging on computational and neural indices of perceptual decision making: A review," Behavioural brain research, vol. 355, pp. 48-55, 2018.

[29] M. Janczyk, P. Mittelstädt, and C. Wienrich's, "Parallel dual-task processing and task-shielding in older and younger adults: Behavioral and diffusion model results," Experimental aging research, vol. 44, no. 2, pp. 95-116, 2018.

[30] G. McKoon and R. Ratcliff, "Aging and iq effects on associative recognition and priming in item recognition," Journal of Memory and Language, vol. 66, no. 3, pp. 416-437, 2012.

[31] R. Ratcliff, A. Thapar, and G. McKoon, "The effects of aging on reaction time in a signal detection task.," Psychology and aging, vol. 16, no. 2, p. 323, 2001. 
[32] R. Ratcliff, P. Gomez, and G. McKoon, "A diffusion model account of the lexical decision task.," Psychological review, vol. 111, no. 1, p. 159, 2004.

[33] A. Thapar, R. Ratcliff, and G. McKoon, "A diffusion model analysis of the effects of aging on letter discrimination.," Psychology and Aging, vol. 18, no. 3, p. 415, 2003.

[34] J. Spaniol, D. J. Madden, and A. Voss, "A diffusion model analysis of adult age differences in episodic and semantic long-term memory retrieval.," Journal of Experimental Psychology: Learning, Memory, and Cognition, vol. 32, no. 1, p. 101, 2006.

[35] J. Spaniol, A. Voss, H. J. Bowen, and C. L. Grady, "Motivational incentives modulate age differences in visual perception.," Psychology and aging, vol. 26, no. 4, p. 932, 2011.

[36] M. von Krause, V. Lerche, A.-L. Schubert, and A. Voss, "Do non-decision times mediate the association between age and intelligence across different content and process domains?," Journal of Intelligence, vol. 8, no. 3, p. 33, 2020.

[37] A.-L. Schubert, D. Hagemann, C. Löffler, and G. T. Frischkorn, "Disentangling the effects of processing speed on the association between age differences and fluid intelligence," Journal of Intelligence, vol. 8, no. 1, p. 1, 2020.

[38] G. McKoon and R. Ratcliff, "Aging and predicting inferences: A diffusion model analysis," Journal of Memory and Language, vol. 68, no. 3, pp. 240-254, 2013.

[39] M. Theisen, V. Lerche, M. von Krause, and A. Voss, "Age differences in diffusion model parameters: a meta-analysis," Psychological Research, pp. 1-10, 2020.

[40] R. Ratcliff and R. Childers, "Individual differences and fitting methods for the two-choice diffusion model of decision making.," Decision, vol. 2, no. 4, p. 237, 2015.

[41] V. Lerche, A. Voss, and M. Nagler, "How many trials are required for parameter estimation in diffusion modeling? a comparison of different optimization criteria," Behavior Research Methods, vol. 49 , no. 2 , pp. 513-537, 2017.

[42] M. D. Lee and E.-J. Wagenmakers, Bayesian cognitive modeling: A practical course. Cambridge university press, 2014.

[43] S. T. Radev, U. K. Mertens, A. Voss, L. Ardizzone, and U. Köthe, "Bayesflow: Learning complex stochastic models with invertible neural networks," IEEE Transactions on Neural Networks and Learning Systems, pp. 1-15, 2020.

[44] K. Xu, B. Nosek, and A. Greenwald, "Psychology data from the race implicit association test on the project implicit demo website," Journal of Open Psychology Data, vol. 2, no. 1, 2014.

[45] R. Ratcliff, "Modeling aging effects on two-choice tasks: Response signal and response time data.," Psychology and Aging, vol. 23, no. 4, p. 900, 2008.

[46] R. Ratcliff, J. Love, C. A. Thompson, and J. E. Opfer, "Children are not like older adults: A diffusion model analysis of developmental changes in speeded responses," Child development, vol. 83 , no. 1 , pp. 367-381, 2012.

[47] P. A. Reuter-Lorenz and D. C. Park, "How does it stac up? revisiting the scaffolding theory of aging and cognition," Neuropsychology review, vol. 24, no. 3, pp. 355-370, 2014.

[48] A. G. Greenwald, D. E. McGhee, and J. L. Schwartz, "Measuring individual differences in implicit cognition: the implicit association test.," Journal of personality and social psychology, vol. 74, no. 6 , p. $1464,1998$.

[49] A. G. Greenwald, B. A. Nosek, and M. R. Banaji, "Understanding and using the implicit association test: I. an improved scoring algorithm.," Journal of personality and social psychology, vol. 85, no. 2, p. 197, 2003. 
[50] M. Usher and J. L. McClelland, "The time course of perceptual choice: the leaky, competing accumulator model.," Psychological review, vol. 108, no. 3, p. 550, 2001.

[51] K. C. Klauer, A. Voss, F. Schmitz, and S. Teige-Mocigemba, "Process components of the implicit association test: A diffusion-model analysis.," Journal of Personality and Social Psychology, vol. 93, no. 3, p. 353, 2007.

[52] D. Matzke and E.-J. Wagenmakers, "Psychological interpretation of the ex-gaussian and shifted wald parameters: A diffusion model analysis," Psychonomic bulletin \& review, vol. 16, no. 5, pp. 798-817, 2009.

[53] B. Bloem-Reddy and Y. W. Teh, "Probabilistic symmetries and invariant neural networks," Journal of Machine Learning Research, vol. 21, no. 90, pp. 1-61, 2020.

[54] D. J. Schad, M. Betancourt, and S. Vasishth, "Toward a principled bayesian workflow in cognitive science.," Psychological Methods, 2020.

[55] J. K. Lindeløv, "mcp: An r package for regression with multiple change points," 2020. 